\title{
The effects of electrode and catalyst selection on microfluidic fuel cell performance
}

\begin{abstract}
A fuel cell can be best defined as an electrochemical converter of fuel and oxidant of chemical energy to electrical energy. The important components of micro fuel cells are the electrodes and catalysts because the kinetics and rates of the electrochemical reactions depend on their materials. All fuel cells consist of two electrodes: the anode, where fuel oxidation takes place, and the cathode, which is used to reduce the oxidants. The present review article highlights the use of a range of electrodes made up of different materials, a variety of catalysts that have been used in previous studies, and their fabrication materials and approaches. In this article, electrodes and catalysts are classified into two types based on the design approach applied to produce the micro fuel cell: micro fuel cell design and conventional assembly design. Most previous studies on fuel cells have demonstrated that the construction and position of the electrodes play crucial roles in improving the performance of micro fuel cells.
\end{abstract}

Keyword: Anode; Cathode; Fuel cells; Microfluidics 